\title{
Evolution of Comovement between Commodity Futures: Do Biofuels Matter?
}

\author{
Ph.D. Candidate Dinçer Dedeoğlu (Bahçeşehir University, Turkey)
}

\begin{abstract}
In this study we investigate the commodity futures linkages for the period 1988:M1-2012:M4. We use monthly futures prices for nine commodities. We employ wavelet analysis to investigate the comovement of commodity futures. By using wavelet based measure of correlation we investigate correlation between commodity futures in both time and frequency domain. The results indicate that correlations are low for short, medium and long-run. We also find evidence of a tendency towards an increase in correlations after 2008. This can be the result of the global crises which has an effect on feedstock costs and the price of energy inputs by putting front a channel through biofuels that links energy and agricultural commodities by increasing the correlation between these commodities after 2008 .
\end{abstract}

Full paper not published upon author's request. 